\title{
COEFFICIENT BOUNDS FOR CERTAIN SUBCLASSES OF M-FOLD SYMMETRIC BI-UNIVALENT FUNCTIONS DEFINED BY CONVOLUTION
}

\author{
F.M. SAKAR, S.M. AYdoĞAN
}

ABstract. In this work, we represent two new subclasses of the bi-univalent functions (by using convolution) which both $f(z)$ and $f^{-1}(z)$ are m-fold symmetric analytic functions. Among other results, for this new subclasses, bounds on the coefficients $\left|a_{m+1}\right|$ and $\left|a_{2 m+1}\right|$ are given in this study.

2010 Mathematics Subject Classification: 30C45.

Keywords: Analytic and univalent functions, Bi-univalent functions, convolution, $m$-fold symmetric functions.

\section{INTRODUCTION}

Let $\mathcal{A}$ indicate an analytic function family, which is normalized under the condition of $f(0)=f^{\prime}(0)-1=0$ in $\mathbb{D}=\{z: z \in \mathbb{C}|z|<1\}$, and are in the form of following equation:

$$
f(z)=z+\sum_{n=2}^{\infty} a_{n} z^{n} .
$$

Furthermore, let $\mathcal{S}$ indicate a subclass in $\mathcal{A}$, being univalent in $\mathbb{D}$ (see [4]).

For $f(z)$ defined by $(1)$ and $\Theta(z)$ defined by

$$
\Theta(z)=z+\sum_{n=2}^{\infty} \Theta_{n} z^{n}, \quad\left(\Theta_{n} \geq 0\right)
$$

the Hadamard product $(f \star \Theta)(z)$ of the functions $f(z)$ and $\Theta(z)$ defined by

$$
(f \star \Theta)(z)=z+\sum_{n=2}^{\infty} a_{n} \Theta_{n} z^{n} .
$$


F.M. Sakar, S.M. Aydoğan - Coefficient bounds for certain subclasses ...

For $0 \leq \beta<1$ and $\lambda \in \mathbb{C}$, we let $Q_{\lambda}(h, \beta)$ be the subclass of $\mathcal{A}$ consisting of functions $f(z)$ of the form (1) and functions $h(z)$ given by

$$
h(z)=z+\sum_{n=2}^{\infty} h_{n} z^{n}, \quad\left(h_{n}>0\right),
$$

and satisfying the analytic criterion:

$\Theta_{\lambda}(h, \beta)=\left\{f \in \mathcal{A}: \Re\left\{(1-\lambda) \frac{(f \star h)(z)}{z}+\lambda(f \star h)^{\prime}(z)\right)>\beta, \quad 0 \leq \beta<1, \quad z \in \mathbb{D}\right\}$.

From the Koebe $1 / 4$ Theorem (for details, see [4]) each univalent $f$ has an inverse $f^{-1}$ fulfilling

$$
f^{-1}(f(z))=z \quad(z \in \mathbb{D})
$$

and

$$
f\left(f^{-1}(w)\right)=w \quad\left(|w|<r_{0}(f), r_{0}(f) \geq \frac{1}{4}\right) .
$$

On the other hand, $f^{-1}$ is represented by

$$
\begin{aligned}
F(w)= & f^{-1}(w)=w-a_{2} w^{2}+\left(2 a_{2}^{2}-a_{3}\right) w^{3}-\left(5 a_{2}^{3}-5 a_{2} a_{3}+a_{4}\right) w^{4}+\cdots \\
& =w+\sum_{n=2}^{\infty} b_{n} w^{n} .
\end{aligned}
$$

When both of $f$ and $f^{-1}$ are univalent, $f \in \mathcal{A}$ is known to be bi-univalent in $\mathbb{D}$. The equation given by (1) shows the class of all bi-univalent functions in $\mathbb{D}$, and this class is represented by $\Sigma$. For detailed information about the class of $\Sigma$ was given in the references [2], [6], [7], [9], [10] and [11].

Let $m \in \mathbb{N}$. A domain $\mathbb{E}$ is known as $m$-fold symmetric if a rotation of $\mathbb{E}$ around origin with an angle $2 \pi / m$ maps $\mathbb{E}$ on itself. It is then seen that, an analytic $f(z)$ in $\mathbb{D}$ being $\mathrm{m}$-fold symmetric $(m \in \mathbb{N})$ satisfies the following condition

$$
f\left(e^{2 \pi i / m} z\right)=e^{2 \pi i / m} f(z) .
$$

Especially, each $f(z)$ and odd $f(z)$ are one-fold symmetric and two-fold symmetric respectively. m-fold symmetric univalent functions in $\mathbb{D}$ are represented by $\mathcal{S}_{m}$. In this case $f \in \mathcal{S}_{m}$ has the following form

$$
f(z)=z+\sum_{n=1}^{\infty} a_{m n+1} z^{m n+1} \quad(z \in \mathbb{D}, m \in \mathbb{N}) .
$$


F.M. Sakar, S.M. Aydoğan - Coefficient bounds for certain subclasses ...

Srivastava et al, in [8] defined m-fold symmetric bi-univalent function. They showed that each $f(z)$ derivatives an $\mathrm{m}$-fold symmetric bi-univalent function for each $(m \in$ $\mathbb{N}$ ) and also they brought out the results of such derivations. In addition, the following expansion of $f^{-1}$ was acquired by them.

$$
\begin{aligned}
F(w) & =w-a_{m+1} w^{m+1}+\left[(m+1) a_{m+1}^{2}-a_{2 m+1}\right] w^{2 m+1} \\
& =-\left[\frac{1}{2}(m+1)(3 m+2) a_{m+1}^{3}-(3 m+2) a_{m+1} a_{2 m+1}+a_{3 m+1}\right] w^{3 m+1}+\cdots
\end{aligned}
$$

where $f^{-1}=F$. We denote by $\Sigma_{m}$ the class of m-fold symmetric bi-univalent functions in $\mathbb{D}$.

A whole treatment of this problem is given in books of several authours [13] by Oldham and Spanier, and [14] by Miller and Ross. However, our study is based on Srivastava [10] who provide more information for the concept of bi-univalent functions and we can find further detailed information in [1]. The object of the present paper is to introduce, by using convolution, new subclasses of the function class bi-univalent functions in which both $f$ and $f^{-1}$ are $m$-fold symmetric analytic functions and obtain coefficient bounds for $\left|a_{m+1}\right|$ and $\left|a_{2 m+1}\right|$ for functions in each of these new subclasses.

\section{Coefficient Estimates for the function Class $\mathcal{H}_{\Sigma_{m}}(\lambda, h, \beta, \alpha)$}

In this section, we introduce by using convolution the function class $\mathcal{H}_{\Sigma_{m}}(\lambda, h, \beta, \alpha)$ by means of the following definition.

Definition 1. A function $f(z) \in \Sigma_{m}$ given by (2) is said to be in the class $\mathcal{H}_{\Sigma_{m}}(\lambda, h, \beta, \alpha)(0<\alpha \leq 1, \lambda \geq 0, m \in \mathbb{N})$ if the following conditions are satisfied:

$$
f \in \Sigma_{m} \text { and }\left|\arg (1-\lambda) \frac{(f \star h)(z)}{z}+\lambda(f \star h)^{\prime}(z)\right|<\frac{\alpha \pi}{2} \quad(z \in \mathbb{U})
$$

and

$$
\left|\arg (1-\lambda) \frac{(f \star h)^{-1}(w)}{w}+\lambda\left((f \star h)^{-1}\right)^{\prime}(w)\right|<\frac{\alpha \pi}{2} \quad(w \in \mathbb{U})
$$

where the function $(f \star h)^{-1}(w)$ defined as follows

$$
(f \star h)^{-1}(w)=w-a_{2} h_{2} w^{2}+\left(2 a_{2}^{2} h_{2}^{2}-a_{3} h_{3}\right) w^{3}-\left(5 a_{2}^{3} h_{2}^{3}-5 a_{2} h_{2} a_{3} h_{3}+a_{4} h_{4}\right) w^{4}+\cdots .
$$


F.M. Sakar, S.M. Aydoğan - Coefficient bounds for certain subclasses ...

We can easily see that for one fold case, when we take $\lambda=1, m=2$ and $h(z)=\frac{z}{1-z}$, the class $\mathcal{H}_{\Sigma}(\lambda, h, \beta)$ reduce to the class $\mathcal{H}_{\Sigma}(\beta)$ studied by Srivastava et al.[8]. So as to calculate our main result, we need to following lemma.

Lemma 1.[12] Let the function $\psi(z)=1+\sum_{k=1}^{\infty} h_{k} z^{k}, z \in \mathbb{U}$, such that $\psi \in$ $P_{m}(\beta)$. Then

$$
\left|h_{k}\right| \leq n(1-\beta), \quad k \geq 1 .
$$

Theorem 1. Let $f \in \mathcal{H}_{\Sigma_{m}}(\lambda, h, \beta, \alpha)(0<\alpha \leq 1, \lambda \geq 0,0 \leq \beta<1, m \in \mathbb{N})$ be given by (3) where the function $h(z)$ is given by (2). If $h_{m+1}, h_{2 m+1} \neq 0$ and $\lambda \in \mathbb{C} \backslash\left\{\frac{-1}{m} ; \frac{-1}{2 m}\right\}$ then

$$
\left|a_{m+1}\right| \leq \min \left\{\sqrt{\frac{2 \alpha^{2} n(1-\beta)}{\left[(1+m \lambda)^{2}+\alpha m\left(1+2 m \lambda-m \lambda^{2}\right)\right]\left|h_{m+1}\right|^{2}}}, \frac{\alpha n(1-\beta)}{(1+m \lambda)\left|h_{m+1}\right|}\right\}
$$

and

$$
\left|a_{2 m+1}\right| \leq \min \left\{\frac{\alpha n(1-\beta)+\frac{\alpha(\alpha-1)}{2} n^{2}(1-\beta)^{2}}{(1+2 m \lambda)\left|h_{2 m+1}\right|}, \frac{\alpha n(1-\beta)}{(1+2 m \lambda)\left|h_{2 m+1}\right|}+\frac{n^{2}(1-\beta)^{2} \alpha^{2}(m+1)}{2(1+m \lambda)^{2}\left|h_{2 m+1}\right|}\right\} .
$$

Proof. Let $f \in \mathcal{H}_{\Sigma_{m}}(\lambda, h, \beta, \alpha)$. Then

$$
(1-\lambda) \frac{(f \star h)(z)}{z}+\lambda(f \star h)^{\prime}(z)=[p(z)]^{\alpha}
$$

and for its inverse map, $(f \star h)^{-1}$, we have

$$
(1-\lambda) \frac{(f \star h)^{-1}(w)}{w}+\lambda\left((f \star h)^{-1}\right)(w)=[q(w)]^{\alpha}
$$

where $p(z), q(w) \in P_{n}(\beta)$. Using the both functions $p(z)$ and $q(w)$ have the following forms.

$$
p(z)=1+p_{m} z^{m}+p_{2 m} z^{2 m}+p_{3 m} z^{3 m}+\cdots
$$

and

$$
q(w)=1+q_{m} w^{m}+q_{2 m} w^{2 m}+q_{3 m} w^{3 m}+\cdots .
$$

Now, equating the coefficients in equation (8) and (9), we get

$$
(1+m \lambda) a_{m+1} h_{m+1}=\alpha p_{m}
$$


F.M. Sakar, S.M. Aydoğan - Coefficient bounds for certain subclasses ...

$$
\begin{gathered}
(1+2 m \lambda) a_{2 m+1} h_{2 m+1}=\alpha p_{2 m}+\frac{\alpha(\alpha-1)}{2} p_{m}^{2} \\
-(1+m \lambda) a_{m+1} h_{m+1}=\alpha q_{m} \\
(1+2 m \lambda)\left[(m+1) a_{m+1}^{2} h_{m+1}^{2}-a_{2 m+1} h_{2 m+1}\right]=\alpha q_{2 m}+\frac{\alpha(\alpha-1)}{2} q_{m}^{2} .
\end{gathered}
$$

Since $p, q \in P_{n}(\beta)$, according to Lemma 1 , the next inequalities hold:

$$
\begin{array}{ll}
\left|p_{t}\right| \leq n(1-\beta), & t \geq 1 \\
\left|q_{t}\right| \leq n(1-\beta), & t \geq 1 .
\end{array}
$$

From (12) and 14, we get

$$
p_{m}=-q_{m}
$$

and

$$
2(1+m \lambda)^{2} a_{m+1}^{2} h_{m+1}^{2}=\alpha^{2}\left(p_{m}^{2}+q_{m}^{2}\right) .
$$

Furthermore from (13), (15) and 22, we have

$$
\begin{aligned}
& a_{m+1}^{2}=\frac{\alpha^{2}\left(p_{2 m}+q_{2 m}\right)}{\left[(1+m \lambda)^{2}+\alpha m\left(1+2 m \lambda-m \lambda^{2}\right)\right]\left|h_{m+1}\right|^{2}} \\
& \leq \frac{2 \alpha^{2} n(1-\beta)}{\left[(1+m \lambda)^{2}+\alpha m\left(1+2 m \lambda-m \lambda^{2}\right)\right]\left|h_{m+1}\right|^{2}} .
\end{aligned}
$$

From (12), by using (16) we get

$$
a_{m+1} \leq \frac{\alpha n(1-\beta)}{(1+m \lambda) h_{m+1}} .
$$

From (13), by using (16) we obtain

$$
a_{2 m+1} \leq \frac{\alpha n(1-\beta)+\frac{\alpha(\alpha-1)}{2} n^{2}(1-\beta)^{2}}{(1+2 m \lambda) h_{2 m+1}} .
$$

Also, substracting (15) from (13), we get

$$
(1+2 m \lambda)\left[2 a_{2 m+1} h_{2 m+1}-(m+1) a_{m+1}^{2} h_{m+1}^{2}\right]=\alpha\left(p_{2 m}-q_{2 m}\right)+\frac{\alpha(\alpha-1)}{2}\left(p_{m}^{2}-q_{m}^{2}\right) .
$$


F.M. Sakar, S.M. Aydoğan - Coefficient bounds for certain subclasses ...

and using (12), (16) and (17) we finally have

$$
(1+2 m \lambda)\left[2 a_{2 m+1} h_{2 m+1}-(m+1) a_{m+1}^{2} h_{m+1}^{2}\right]=\alpha\left(p_{2 m}-q_{2 m}\right)+\frac{\alpha(\alpha-1)}{2}\left(p_{m}^{2}-q_{m}^{2}\right) .
$$

From (22) we can obtain the equality given as

$$
a_{m+1}^{2} h_{m+1}^{2}=\frac{\alpha^{2}\left(p_{m}^{2}+q_{m}^{2}\right)}{2(1+m \lambda)^{2}}
$$

when we write this equality given as (23) in (22) and by using (16) and (17), and also observing that $p_{m}^{2}=q_{m}^{2}$, it follows that

$$
\begin{gathered}
a_{2 m+1}=\frac{\alpha\left(p_{2 m}-q_{2 m}\right)+\frac{\alpha(\alpha-1)}{2}\left(p_{m}^{2}-q_{m}^{2}\right)+(1+2 m \lambda)(m+1) \frac{\alpha^{2}\left(p_{m}^{2}+q_{m}^{2}\right)}{2(1+m \lambda)^{2}}}{2(1+2 m \lambda) h_{2 m+1}} . \\
a_{2 m+1}=\frac{\alpha\left(p_{2 m}-q_{2 m}\right)}{2(1+2 m \lambda) h_{2 m+1}}+\frac{\alpha(\alpha-1)\left(p_{m}^{2}-q_{m}^{2}\right)}{4(1+2 m \lambda) h_{2 m+1}}+\frac{(m+1) \alpha^{2}\left(p_{m}^{2}+q_{m}^{2}\right)}{4(1+m \lambda)^{2} h_{2 m+1}}
\end{gathered}
$$

Taking the absolute value of (24) and applying (16) and (17), and also taking into consideration that $p_{m}^{2}=q_{m}^{2}$, we obtain

$$
\left|a_{2 m+1}\right| \leq \frac{\alpha n(1-\beta)}{(1+2 m \lambda)\left|h_{2 m+1}\right|}+\frac{n^{2}(1-\beta)^{2} \alpha^{2}(m+1)}{2(1+m \lambda)^{2}\left|h_{2 m+1}\right|} .
$$

which completes the proof of the Theorem 1.

\section{Coefficient Estimates for the function Class $\mathcal{H}_{\Sigma_{m}}(\lambda, h, \xi)$}

Definition 2. A function $f(z) \in \Sigma_{m}$ given by (3) is said to be in the class $\mathcal{H}_{\Sigma_{m}}(\lambda, h, \xi)(0 \leq \xi<1, \lambda \geq 0, m \in \mathbb{N})$ if the following conditions are satisfied:

$$
f \in \Sigma_{m} \text { and } \operatorname{Re}\left\{(1-\lambda) \frac{(f \star h)(z)}{z}+\lambda(f \star h)^{\prime}(z)\right\}>\xi(z \in \mathbb{U})
$$

and

$$
\operatorname{Re}\left\{(1-\lambda) \frac{(f \star h)^{-1}(w)}{w}+\lambda\left((f \star h)^{-1}\right)^{\prime}(w)\right\}>\xi(w \in \mathbb{U})
$$

where the function $(f \star h)^{-1}(w)$ defined as follows

$$
(f \star h)^{-1}(w)=w-a_{2} h_{2} w^{2}+\left(2 a_{2}^{2} h_{2}^{2}-a_{3} h_{3}\right) w^{3}-\left(5 a_{2}^{3} h_{2}^{3}-5 a_{2} h_{2} a_{3} h_{3}+a_{4} h_{4}\right) w^{4}+\cdots .
$$


F.M. Sakar, S.M. Aydoğan - Coefficient bounds for certain subclasses ...

Theorem 2. Let $f \in \mathcal{H}_{\Sigma_{m}}(\lambda, h, \xi)(\lambda \geq 0,0 \leq \xi<1, m \in \mathbb{N})$ be given by (3) where the function $h(z)$ is given by (2). If $h_{m+1}, h_{2 m+1} \neq 0$ and $\lambda \in \mathbb{C} \backslash\left\{\frac{-1}{m} ; \frac{-1}{2 m}\right\}$ then

$$
\left|a_{m+1}\right| \leq \sqrt{\frac{2 n(1-\xi)}{(m+1)(1+2 m \lambda)\left|h_{m+1}\right|^{2}}}
$$

and

$$
\left|a_{2 m+1}\right| \leq \frac{n(1-\xi)}{(1+2 m \lambda)\left|h_{2 m+1}\right|}+\frac{(m+1) n^{2}(1-\xi)^{2}}{2(1+m \lambda)^{2}\left|h_{2 m+1}\right|} .
$$

Proof.Let $f \in \mathcal{H}_{\Sigma_{m}}(\lambda, h, \xi)$. From the Definition 2 we obtain

$$
(1-\lambda) \frac{(f \star h)(z)}{z}+\lambda(f \star h)^{\prime}(z)=p(z)
$$

and

$$
(1-\lambda) \frac{(f \star h)^{-1}(w)}{w}+\lambda\left((f \star h)^{-1}\right)^{\prime}(w)=q(w)
$$

where $p(z), q(w) \in P_{n}(\xi)$.

Using the fact that $p(z), q(w)$ have the Taylor expansions given by (10) and (11), respectively. Equating coefficients (30) and (31) yields

$$
\begin{gathered}
(1+m \lambda) a_{m+1} h_{m+1}=p_{m} \\
(1+2 m \lambda) a_{2 m+1} h_{2 m+1}=p_{2 m} \\
-(1+m \lambda) a_{m+1} h_{m+1}=q_{m} \\
(1+2 m \lambda)\left[(m+1) a_{m+1}^{2} h_{m+1}^{2}-a_{2 m+1} h_{2 m+1}\right]=q_{2 m} .
\end{gathered}
$$

Since $p(z), q(w) \in P_{n}(\xi)$, with respect to Lemma 1, the inequalities given (16) and (17) and thus, from (33) and (35), by using the inequalities (16) and (17) we get

$\left|a_{m+1}\right|^{2} \leq \frac{\left(\left|p_{2 m}\right|+\left|q_{2 m}\right|\right)(1-\xi)}{(m+1)|(1+2 m \lambda)|\left|h_{m+1}\right|^{2}} \leq \frac{2 n(1-\xi)}{(m+1)|(1+2 m \lambda)|\left|h_{m+1}\right|^{2}}$, for $\lambda \in \mathbb{C} \backslash\left\{\frac{-1}{2 m}\right\}$

From (32), by using (16) we obtain 
F.M. Sakar, S.M. Aydoğan - Coefficient bounds for certain subclasses ...

$$
a_{m+1} \leq \frac{n(1-\xi)}{|(1+m \lambda)|\left|h_{m+1}\right|} \text { for } \lambda \in \mathbb{C} \backslash\left\{\frac{-1}{m}\right\} .
$$

Also from (33) and by using (16) we obtain

$$
a_{2 m+1} \leq \frac{n(1-\xi)}{|(1+2 m \lambda)|\left|h_{2 m+1}\right|} \text { for } \lambda \in \mathbb{C} \backslash\left\{\frac{-1}{2 m}\right\} .
$$

Also, subtracting (13)from (33), we obtain

$$
(1+2 m \lambda)\left[2 a_{2 m+1} h_{2 m+1}-(m+1) a_{m+1}^{2} h_{m+1}^{2}\right]=p_{2 m}-q_{2 m},
$$

and using (32), (16) and (17) in the above equality, we have

$$
\left|a_{2 m+1}\right| \leq \frac{n(1-\xi)}{|(1+2 m \lambda)|\left|h_{2 m+1}\right|}+\frac{(m+1) n^{2}(1-\xi)^{2}}{2\left|(1+m \lambda)^{2}\right|\left|h_{2 m+1}\right|} \quad \text { for } \quad \lambda \in \mathbb{C} \backslash\left\{\frac{-1}{m} ; \frac{-1}{2 m}\right\}
$$

which completes the proof of Theorem 2 .

Taking some special values of parameters we can obtain some corollories given below.

Corollary 1. Taking $\lambda=0$ in Theorem 1 we obtain

$$
\left|a_{m+1}\right| \leq \min \left\{\sqrt{\frac{2 \alpha^{2} n(1-\beta)}{(1+\alpha m)\left|h_{m+1}\right|^{2}}}, \frac{\alpha n(1-\beta)}{\left|h_{m+1}\right|}\right\} \text { for } h_{2 m+1} \neq 0
$$

and

$$
\left|a_{2 m+1}\right| \leq \min \left\{\frac{\alpha n(1-\beta)+\frac{\alpha(\alpha-1)}{2} n^{2}(1-\beta)^{2}}{\left|h_{2 m+1}\right|}, \frac{\alpha n(1-\beta)}{\left|h_{2 m+1}\right|}+\frac{n^{2}(1-\beta)^{2} \alpha^{2}(m+1)}{2\left|h_{2 m+1}\right|}\right\} \text {, for } h_{2 m+1} \neq 0 .
$$

Corollary 2. Taking $\lambda=1$ Theorem 1 , we obtain

$\left|a_{m+1}\right| \leq \min \left\{\sqrt{\frac{2 \alpha^{2} n(1-\beta)}{\left[(1+m)^{2}+\alpha m(1+m)\right]\left|h_{m+1}\right|^{2}}}, \frac{\alpha n(1-\beta)}{(1+m)\left|h_{m+1}\right|}\right\}$ for $h_{2 m+1} \neq 0$

and

$\left|a_{2 m+1}\right| \leq \min \left\{\frac{\alpha n(1-\beta)+\frac{\alpha(\alpha-1)}{2} n^{2}(1-\beta)^{2}}{(1+2 m)\left|h_{2 m+1}\right|}, \frac{\alpha n(1-\beta)}{(1+2 m)\left|h_{2 m+1}\right|}+\frac{n^{2}(1-\beta)^{2} \alpha^{2}(m+1)}{2(1+m)^{2}\left|h_{2 m+1}\right|}\right\}$,

for $h_{2 m+1} \neq 0$. 
F.M. Sakar, S.M. Aydoğan - Coefficient bounds for certain subclasses ...

When we put $\lambda=1, n=2$ and $h(z)=\frac{z}{1-z}$ in Theorem 1 we can easily obtain the next Corollary. Corollary 3. For $f \in H_{\Sigma}(\beta)$ we obtain next inequalities

$$
\left|a_{m+1}\right| \leq \min \left\{\sqrt{\frac{4 \alpha^{2}(1-\beta)}{(1+m)^{2}+\alpha m(1+m)}}, \frac{2 \alpha(1-\beta)}{(1+m)}\right\}
$$

and

$$
\left|a_{2 m+1}\right| \leq \min \left\{\frac{2 \alpha(1-\beta)+\frac{4 \alpha(\alpha-1)(1-\beta)^{2}}{2}}{(1+2 m)}, \frac{2 \alpha(1-\beta)}{(1+2 m)}+\frac{4(1-\beta)^{2} \alpha^{2}(m+1)}{2(1+m)^{2}}\right\} .
$$

For one-fold case and $\alpha=1$ in Corollory 5 the last Corollary is obtained as follows:

Corollary 4. For one-fold case and $\alpha=1$ in Corollory 3 the last Corollary is obtained as follows [3]:

For $f \in H_{\Sigma}(\beta)$ we obtain next inequalities

$$
\left|a_{2}\right| \leq\left\{\sqrt{\frac{2(1-\beta)}{3}}\right\} \quad 0 \leq \beta \leq \frac{1}{3}
$$

and

$$
\begin{gathered}
\left|a_{3}\right| \leq \frac{2(1-\beta)}{3} \quad 0 \leq \beta \leq \frac{1}{3} \\
\left|2 a_{2}^{2}-a_{3}\right| \leq \frac{2(1-\beta)}{3} \quad 0 \leq \beta \leq \frac{1}{3} .
\end{gathered}
$$

Remark. For 1-fold symmetric bi-univalent functions, Theorem 1 and Theorem 2 reduce to results given by Frasin and Aouf [5]. Also,for 1-fold symmetric biunivalent functions, if we put $\lambda=1$ in our Theorems, we obtain to results which were given by Srivastava et al.[8]. Furthermore, for $m$-fold symmetric bi-univalent functions, if we put $\lambda=1$ in Theorem 1 and Theorem 2, we obtain to results which were given by Srivastava et al.[8].

Acknowledgement. The work presented here is supported by Batman University Scientific Research Project Coordination Unit. Project Number: BTUBAP2018-IIBF-2.

\section{REFERENCES}

[1] A. Akgül, On the coefficient estimates of analytic and bi-univalent m-fold symmetric functions, Mathematica Aeterna, 7(3) (2017), 253-260. 
F.M. Sakar, S.M. Aydoğan - Coefficient bounds for certain subclasses ...

[2] A. Akgül, Ş. Altınkaya, Coefficient estimates associated with a new subclass of bi-univalent functions, Acta Universitatis Apulensis, 52 (2017), 121-128.

[3] Ş. Altınkaya, S. Yalçın Coefficient problem for certain subclasses of bi-univalent functions defined by convolution, Mathematica Moravica 20, 2 (2016), 1521.

[4] P.L. Duren Univalent Functions, In: Grundlehren der Mathematischen Wissenschaften, Band 259, New York, Berlin, Heidelberg and Tokyo, Springer-Verlag, 1983.

[5] B.A. Frasin, M.K. Aouf, New subclasses of bi-univalent functions. Applied Mathematics Letters 24 (2011), 1569-1573.

[6] M. Lewin On a coefficient problem for bi-univalent functions, Proc. Amer. Math. Soc. 18 (1967), 63-68.

[7] M.E. Netanyahu The minimal distance of the image boundary from the origin and the second coefficient of a univalent function in $|z|<1$. Arch. Rational Mech. Anal. 32 (1969), 100-112.

[8] H.M. Srivastava, S. Sivasubramanian, R. Sivakumar Initial coefficient bounds for a subclass of $m$-fold symmetric bi-univalent functions, Tbilisi Mathematical Journal 7 (2014), 1-10.

[9] T.S. Taha, Topics in Univalent Function Theory, Ph.D. Thesis, University of London, London, UK, 1981.

[10] H.M. Srivastava, A.K. Mishra, P. Gochhayat Certain subclasses of analytic and bi-univalent functions, Applied Mathematics Letters 23, 10 (2010), 1188-1192.

[11] D.A. Brannan, T.S. Taha On some classes of bi-univalent functions, in: Mathematical Analysis and Its Applications(Mazhar, S. M., Hamoui, A. and Faour, N. S. Editors) (Kuwait; February 18-21, 1985), KFAS Proceedings Series, Vol. 3, Pergamon Press (Elsevier Science Limited), Oxford, 1988, pp. 53-60; see also Studia Univ. Babeş-Bolyai Math. 31, 1 (1986), 70-77.

[12] P. Goswami, B.S. Alkahtani, T. Bulboacă, Estimate for Initial Maclaurin Coefficients of Certain Sublasses of Bi-univalent Functions, http://arxiv.org/abs/1503.04644v1, 2015.

[13] K.B. Oldham, J. Spanier, The Fractional Calculus, Academic Press 1974.

[14] K.S. Miller, B. Ross, An Introduction to the Fractional Calculus and Fractional Differential Equations, John Wiley and Sond, Inc.1993 New York.

F. Müge Sakar

Department of Mathematics, Faculty of Economics and Administrative Sciences, Batman University 
F.M. Sakar, S.M. Aydoğan - Coefficient bounds for certain subclasses ...

Batman, Turkey

email: mugesakar@hotmail.com(Corresponding author)

S. Melike Aydoğan

Department of Mathematics, Faculty of Science and Letters, Istanbul Technical University,

Istanbul, Turkey

email: melikeaydogan.itu@gmail.com 